\title{
IL-3-producing basophils are required to exacerbate airway hyperresponsiveness in a murine inflammatory model
}

\author{
R. Rignault-Bricard ${ }^{1,2}$ | F. Machavoine ${ }^{1}$ | S. Mecheri ${ }^{3}$ | O. Hermine ${ }^{2,4}$ | E. Schneider ${ }^{1}$ | \\ M. Dy ${ }^{1}$ | M. Leite-de-Moraes ${ }^{1}$ (D)
}

${ }^{1}$ Laboratory of Immunoregulation and Immunopathology, Institut Necker-Enfants Malades, Centre National de la Recherche Scientifique (CNRS), Unité Mixte de Recherche (UMR) 8253, Institut National de la Santé et de la Recherche Médicale (Inserm) UMR 1151, Université Paris Descartes Sorbonne Paris Cité, Paris, France

${ }^{2}$ Institut Imagine Inserm UMR1163, CNRS Equipe de Recherche Labelisée (ERL) 8654, Université Paris Descartes Paris, Paris, France

${ }^{3}$ Institut Pasteur, Unité de Biologie des Interactions Hôte Parasites, CNRS ERL9195, Inserm UMR1201, Paris, France

${ }^{4}$ Department of Hematology, Hôpital Necker Enfants Malades, Assistance Publique Hôpitaux de Paris, Université Paris Descartes Paris, Paris, France

\section{Correspondence}

Maria Leite-de-Moraes, Laboratory of Immunoregulation and Immunopathology, Institut Necker-Enfants Malades, CNRS UMR 8253, INSERM UMR 1151, Université Paris Descartes Sorbonne Paris Cité, Paris, France. Email: maria.leite-de-moraes@parisdescartes.fr

Funding information

CNRS; INSERM; Université Paris Descartes; Fondation Day Solvay

\begin{abstract}
Background: Basophils are commonly associated with allergic responses because of their ability to produce large amounts of pro-Th2 2 cytokines and histamine. However, the mechanisms through which bone marrow-resident basophils (BMRB) become fully competent cytokine and histamine producers in response to IgE crosslinking are poorly understood. Here, we sought to determine the role of IL- 3 in promoting pro-Th2 basophils.

Methods: BMRB and basophils exposed to IL-3 in vitro and in vivo were evaluated for their production of Th2 cytokines and histamine in response to FceRI crosslinking on both protein and gene expression levels. In vivo relevance of our findings was assessed in a model of ovalbumin-induced allergic asthma using IL-3-deficient and wild-type mice in a protocol of adoptive basophil transfer.

Results: We show that BMRB and basophils previously exposed to IL-3 differ in their ability to generate cytokines (IL-4, IL-6, IL-13, and GM-CSF) and histamine in response to Fc\&RI crosslinking, reflecting two stages of maturation. Exposure to IL-3 initiated an autocrine loop of endogenous IL-3 production that enhanced histamine and cytokine production upon FceRI crosslinking. This increased responsiveness required calcium flux and was dependent on calcineurin and store-operated calcium channels. Our findings are of pathophysiological relevance, as assessed by the failure of IL-3-deficient mice to develop airway hyperreactivity, which could be restored by adoptive transfer of IL-3-derived basophils recovered from wild-type mice.

Conclusion: IL-3-dependent basophils promote Th2 allergic AHR, which designates the IL-3/basophil axis as a promising therapeutic target for the treatment of basophil-dependent asthma.

KEYWORDS

asthma, basophils, calcium, histamine, IL-3
\end{abstract}




\section{1 | INTRODUCTION}

Basophils are the least abundant granulocytes, making up less than $1 \%$ of peripheral blood leukocytes. Their activities as pro-inflammatory effector cells represent a double-edged sword, deleterious, by contributing to allergic disorders, and beneficial, by providing protection against helminth infections. ${ }^{1-5}$ Basophils are involved in the development of Th2-type immune responses, mainly as a source of large amounts of IL-4, generated in response to a variety of stimuli dependent or not on IgE. ${ }^{1,6-9}$ On a per cell basis, basophils are the most efficient granulocytic IL-4 producers. ${ }^{10-13}$ They play a crucial role in eosinophilic lung inflammation induced by protease allergens, as their deletion impairs airway eosinophilia, mucus formation, and AHR (airway hyperresponsiveness) development in the papain model. ${ }^{14}$ Further, they strongly express the high-affinity lgE receptor and respond to its crosslinking by producing histamine, which, in turn, takes part in the regulation of immune responses and controls its own synthesis as well as that of IL-4, IL-6, and IL-13, produced concomitantly. ${ }^{15,16}$

Basophils arise from hematopoietic stem cells and are believed to terminate their differentiation in the bone marrow before entering the circulation as fully matured cells. ${ }^{17}$ However, the mechanisms underlying this process are not completely understood. IL-3 is allegedly the most potent growth factor for basophils, but it is not essential for their generation under physiological conditions as their incidence in the bone marrow is not impaired in IL-3-deficient mice. ${ }^{18}$ Thymic stromal lymphopoietin (TSLP) at high concentrations can mimic the proliferative effect of IL-3 on basophils ${ }^{19}$ and target the population residing in the bone marrow directly to elicit a population that promotes type 2 inflammation. ${ }^{20}$ However, it seems that this effect required the presence of IL-3. Further, human basophils from allergic patients did not respond to TSLP. ${ }^{21}$ Taken together, these findings support a role of IL-3 in the differentiation of pro-Th2 basophils but leave open the question of its specific functions affecting basophils in both physiological and pathophysiological allergic conditions.

To address this issue, we compared the functional characteristics of resident basophils, sorted ex vivo from freshly isolated bone marrow cells (bone marrow-resident basophils or BMRB) with those of basophils having been exposed to IL-3 either in vitro during bone marrow culture or in vivo upon injection of IL-3. We found that basophils exposed to IL-3 responded more efficiently to Fc\&RI stimulation, which increased histamine and cytokine synthesis, while BMRB were less reactive, particularly in terms of cytokine production. Starting from this finding, we set out to examine the mechanisms through which these differences emerged and attempted to identify the molecules involved, their mode of action, as well as the in vivo relevance in an allergic asthma model.

\section{2 | MATERIALS AND METHODS}

A detailed description of the methods is provided in online supplement Appendix S1.

\subsection{Mice}

Eight- to ten-week-old specific pathogen-free C57BL/6J, BALB/c, C57BL/10 IL-3-deficient (IL-3KO), and C57BL/10 wild-type (WT) mice were bred in our facility or purchased from Janvier Labs. All animal experiments were approved by the French Institutional Committee (APAFIS\#4105-201511171831592).

\section{IL-3-dependent basophils are required to exacerbate Th2 allergic AHR}

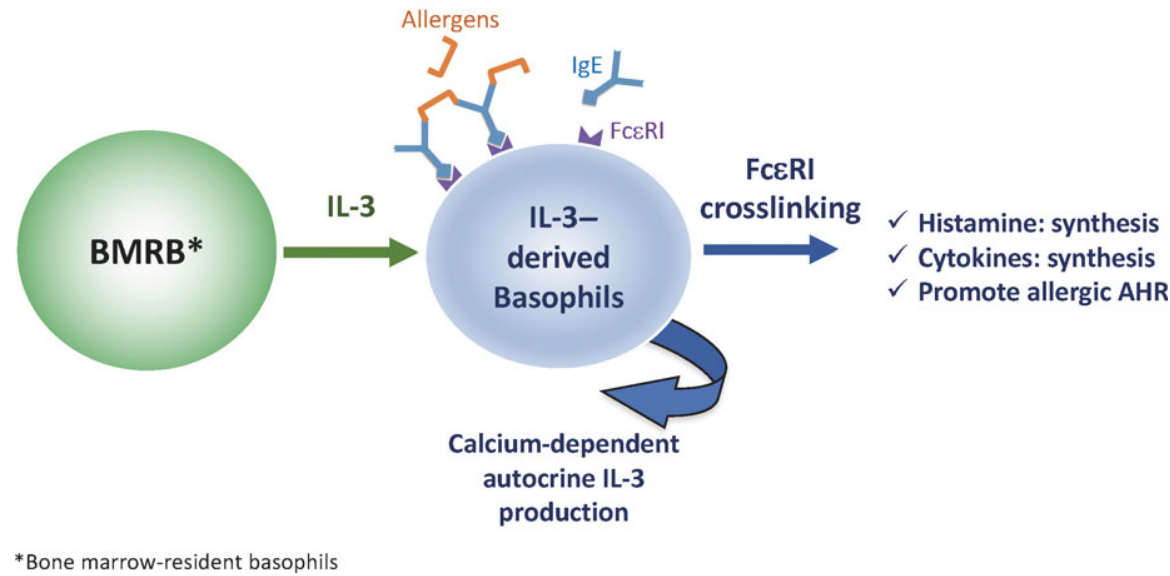

\section{GRAPHICAL ABSTRACT}

FcERI crosslinking on IL-3-derived basophils induces concomitant histamine and cytokine synthesis depending on calcineurin and store-operated calcium entry. Autocrine IL-3 is essential for enhancing the responsiveness of bone marrow basophils to Fc\&R1 crosslinking. IL-3-deficient mice are unable to develop asthmatic hyperreactivity, which can be fully restored by the adoptive transfer of IL-3-producing basophils. 


\subsection{Culture and sorting of basophils}

Basophil-enriched populations were generated from total bone marrow cells cultured for 8 days with IL-3 ( $1 \mathrm{ng} / \mathrm{mL}$ ) (R\&D Systems), as described before. ${ }^{22}$ They contained on average $25 \%-40 \%$ basophils defined as c-kit ${ }^{-} \mathrm{Fc} \varepsilon \mathrm{RI}^{+} \mathrm{CD} 49 \mathrm{~b}^{+}$cells. The $\mathrm{CD} 49 \mathrm{~b}^{+}$subset was sorted by magnetic positive selection using a RoboSep automaton (StemCell Technologies). Sorted cells were $98 \% \mathrm{Fc \varepsilon RI}^{+} \mathrm{c}-\mathrm{kit}^{-}$upon reanalysis. BMRB were enriched from freshly isolated bone marrow cells by depleting NK1.1 $1^{+}$cells, followed by positive selection of the CD49b subset and electronic sorting of $\mathrm{c}^{-} \mathrm{kit}^{-} \mathrm{CD} 11 \mathrm{~b}^{+}$population using a FACSAria II cell sorter (BD Biosciences). Purity was $>98 \%$ upon reanalysis of aliquots stained with anti-FceRl $\alpha \mathrm{mAb}$. Sorted basophils were incubated at a concentration of $25 \times 10^{4}$ per $\mathrm{ml}$ in the presence of $10 \mathrm{ng} / \mathrm{mL} \mathrm{IL}-3$ or ionomycin (Sigma-Aldrich) $\left(10^{-6} \mathrm{M}\right)$ for 4 or 20 hours. For Fc\&RI crosslinking, basophils were sensitized with the monomeric cytokinergic IgE anti-DNP (clone SPE-7) (Sigma-Aldrich) at $5 \mu \mathrm{g} / \mathrm{mL}$, with the IgE anti-DNP specific IgE mAb (obtained from hybridoma H1-DNP- $\varepsilon-26.82$ supernatants) plus DNP-HSA conjugate (Biotrend Chemikalien) $(1 \mu \mathrm{g} / \mathrm{mL})$ or with the anti-Fc\&RI mAb (clone MAR-1) $(10 \mathrm{ng} / \mathrm{mL})$ during 4 hours or 24 hours at $37^{\circ} \mathrm{C}$. Histamine and cytokines were measured in supernatants as previously described. ${ }^{22}$ Alpha-fluoro-methylhistidine $(\alpha \mathrm{FMH})$, FK506, and GSK$7975 \mathrm{~A}$, used at a final concentration of $5 \times 10^{-5} \mathrm{~mol} / \mathrm{L}, 10^{-6} \mathrm{~mol} / \mathrm{L}$, and $10^{-5} \mathrm{~mol} / \mathrm{L}$, respectively, were added to cell cultures 30 minutes before stimulation. These compounds were not toxic at the dose used. Cell viability was evaluated by the XTT-derived assay (Promega) after incubation, according to the supplier's recommendations. The information of reagents and suppliers is included in Table S1.

\section{3 | In vivo IL-3 plus anti-IL-3 treatment}

Mice received an intraperitoneal injection of IL-3 (10 $\mu \mathrm{g} /$ mouse) (R\&D Systems) mixed with anti-IL-3 (5 $\mu \mathrm{g} /$ mouse) (BD Biosciences), 4 days before killing. Bone marrow, spleen, and liver leukocytes were isolated as previously described ${ }^{22,23}$ or as detailed below for lung leukocytes.

\section{4 | Flow cytometry}

Fresh or cultured basophils were stained and analyzed by flow cytometry, as described. ${ }^{25}$ The following appropriately labeled mAbs were used anti: CD117(c-kit), CD11b, CD69, CD200R3, CD49b, FceRla. Fixable viability dye was used to exclude dead cells. For intracellular staining, cells were fixed, permeabilized, and then incubated with anti-: IL-3, IL-4, IL-13 mAbs. Cells were then washed, acquired on a FACSCanto II or Fortessa (BD Biosciences), and analyzed using FlowJo software. Further information on antibodies and fluorochromes used is included in Table S2.

\section{5 | Airway allergen sensitization and challenge model}

$\mathrm{BALB} / \mathrm{c}$ (the main mouse strain used to this type of protocol), IL$3 \mathrm{KO}$, and littermate control mice were immunized by i.p. injection of
$100 \mu \mathrm{g}$ OVA (Sigma-Aldrich) adsorbed on $1.5 \mathrm{mg}$ alum adjuvant (Merck) in $0.4 \mathrm{~mL}$ saline solution, then challenged on 3 consecutive days (D7, D8, D9) with intranasal OVA (50 $\mu \mathrm{g} /$ mouse) or saline solution. In some experiments, $75 \times 10^{3}$ sorted basophils were adoptively transferred intravenously to immunized mice $1 \mathrm{~h}$ before the first challenge. Twenty-four hours after the last challenge, FlexiVent apparatus (SCIREQ) was used to access airway-specific resistance (Rn, tidal volume of $10 \mathrm{~mL} / \mathrm{kg}$ at a respiratory rate of 150 breath/ $\min$ in response to increasing doses of aerosolized acetyl- $\beta$-methacholine chloride (methacholine; Sigma-Aldrich). Assessments were performed at least three times, and the maximum $R$-value obtained after each dose of methacholine was used for the measure. Airway inflammation was assessed in cytospin preparations of cells in bronchoalveolar lavage fluid (BALF) that were stained with MayGrünwald/Giemsa (Merck).

\section{6 | Statistics}

Data are expressed as means \pm SEM. The AHR values were analyzed with repeated-measures 2-way ANOVA followed by the Bonferroni correction as a post hoc test. All other values were analyzed with Mann-Whitney $U$ test. Results were considered significant at a $P$ value of .05 or less $(* P<.05 ; * * P<.01$; $* * * P<.001)$. Data were analyzed using GraphPad Prism version 6 (GraphPad Software).

\section{\begin{tabular}{l|l}
3 & RESULTS
\end{tabular}}

\subsection{FceRI crosslinking on basophils cultured with IL-3 induces concomitant histamine and cytokine synthesis depending on calcineurin and store- operated calcium entry}

FceRI crosslinking, whether in response to DNP/anti-DNP IgE (clone H1-ع-26.82), monomeric cytokinergic SPE-7 IgE, or anti-FceRI mAb,

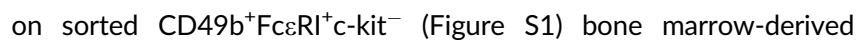
basophils obtained after 8 days of culture with IL-3 (dubbed IL-3derived basophils), generated large amounts of histamine (Figure 1A). This production resulted from neosynthesis rather than classical degranulation for the following reasons: 1 /the relatively low, initial intracellular histamine content in bone marrow-derived basophils $\left(0.30 \pm 0.04 \mu \mathrm{g} / 10^{6}\right.$ cells), which increased slightly over the incubation period, cannot account for the high concentrations in supernatants after 4 (Figure S2A) or 20 hours (Figure S2B) of Fc\&RI stimulation; 2/histamine failed to increase in the presence of $\alpha F M H$ (Figure 1A), the specific inhibitor of histidine decarboxylase (HDC), the unique histamine-forming enzyme (Figure 1A); and 3/FceRI crosslinking promoted increased HDC mRNA transcription, as assessed by quantitative RT-PCR (Figure S2C). In all cases, FceRIinduced histamine synthesis occurred together with the production of IL-4, IL-6, IL-13, and GM-CSF (Figure 1B).

Intracellular $\mathrm{Ca}^{2+}$ signaling was required for this activity, as assessed by its abrogation in the presence of the calcineurin inhibitor 
FIGURE 1 Histamine and cytokine synthesis by stimulated IL-3-derived basophils. A, Histamine (HA) was measured in supernatants from basophils from C57BL/6J mice following FceRI crosslinking (SPE-7, DNP/anti-DNP IgE, or anti-FcERI) in the presence or absence of the HDC inhibitor $\alpha \mathrm{FMH}(\mathrm{n}=3)$. B, Cytokine production following Fc\&RI stimulation with SPE-7 or DNP/anti-DNP IgE $(n>5)$. ( $C$ and $D)$, The effect of the ORAI1 inhibitor (GSK-7975A) on HA and cytokine synthesis by IL-3-derived basophils following ionomycin (C) or Fc\&RI crosslinking (D) was assessed $(n=5)$. $P$ values were determined between $w / o$ and with GSK-7975A samples
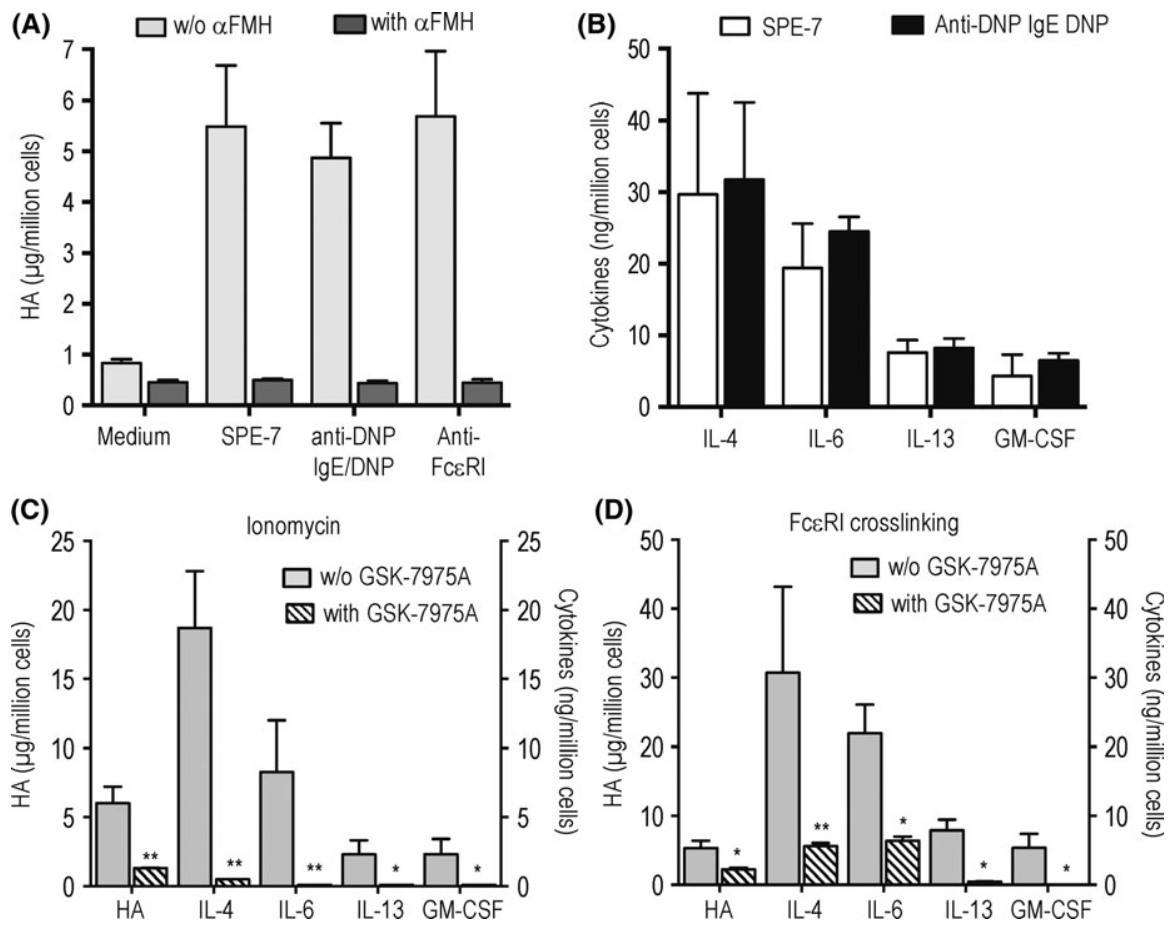

FK506 $6^{26,27}$ (Table S3). In accordance with this conclusion, calcium ionophores such as ionomycin or release of $\mathrm{Ca}^{2+}$ stores from the endoplasmic reticulum (ER) by thapsigargin ${ }^{28}$ mimicked the effect of Fc\&RI stimulation on histamine and cytokine synthesis (Figure S2D) and were likewise inhibited by FK506, as shown in Table S3 for ionomycin.

Thapsigargin-induced calcium release from the ER is usually followed by the opening of store-operated $\mathrm{Ca}^{2+}$ channels (SOCs) to replenish the stores. ${ }^{29}$ IL-3-derived basophils are competent for this pathway as they expressed mRNA encoding ORAI1 ${ }^{30}$ (a key subunit of SOCs) (Figure S2E). The involvement of this mechanism of action was confirmed by the almost complete inhibition of ionomycin- and Fc\&RI crosslinking-induced histamine and cytokine synthesis in IL-3derived basophils exposed to the ORAI1 inhibitor GSK-7975A $\mathrm{A}^{31-33}$ (Figure 1C,D). Collectively, these results suggest that SOCs play an important role in cytokine and histamine synthesis by IL-3-derived basophils.

\section{2 | IL-3-derived basophils respond to Fc\&RI crosslinking by calcium-dependent production of endogenous IL- 3 that enhances their histamine and cytokine synthesis}

It is noteworthy that IL-3-derived basophils themselves produced IL3 upon Fc\&RI crosslinking, as assessed by both protein and mRNA analyses (Figure 2A,B). This autocrine IL-3 production depended on calcium, as it failed to occur in the presence of the calcineurin inhibitor FK506 and was mimicked by ionomycin (Figure 2A,B). Furthermore, inhibition by the ORAI1 inhibitor GSK-7975A proved the involvement of SOCs (Figure 2A,B). Endogenously produced IL-3 might actually account for the increased survival of IL-3-derived basophils following Fc\&RI crosslinking, relative to their IL-3-deficient counterpart stimulated in the same conditions (Figure 2C). As shown in (Figure 2D,E), basophils from IL-3KO mice produced also less histamine and cytokine in response to Fc\&RI crosslinking, as did WT IL3-derived basophils exposed to anti-IL-3 (Figure S3A). Finally, the addition of IL-3 during FceRI crosslinking on IL-3-derived basophils from IL-3KO mice enhanced their ability to produce histamine and cytokines (Figure 2F) as well as their survival (Figure 2G), while no significant effect was observed when IL-3-derived basophils from WT mice were used instead (Figure 2G, Figure S3B). Overall, these data support the conclusion that endogenous IL-3 contributes to the activation as well as the survival of IL-3-derived basophils in response to Fc\&RI crosslinking.

\section{3 | BMRB are less reactive to Fc\&RI crosslinking than IL-3-derived basophils}

Similarly to IL-3-derived basophils, ex vivo electronically sorted CD49b ${ }^{+} \mathrm{FcERI}^{+} \mathrm{c}$-kit ${ }^{-} \mathrm{CD} 11 \mathrm{~b}^{+}$bone marrow-resident basophils (BMRB) secreted cytokines together with histamine when activated through Fc\&RI (Figure 3A). However, we found striking quantitative differences as ex vivo BMRB generated consistently less histamine and cytokines than their culture-derived counterpart (Figure 3A). This was particularly true for IL-13 and GM-CSF, which were undetectable in supernatants from BMRB. The increased responsiveness of IL-3-derived basophils to ligands of Fc\&RI coincided with its upregulation and that of the activation markers CD200R3 and CD69 (Figure S4).

Furthermore, the cytokine pattern generated in response to IL-3 differed strikingly between the two cell populations as freshly isolated basophils produced more IL- 4 and IL- 6 than those that had encountered the growth factor during their expansion in vitro 


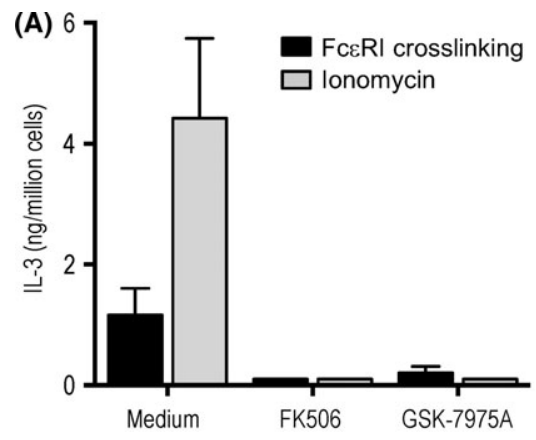

(C)

(D)

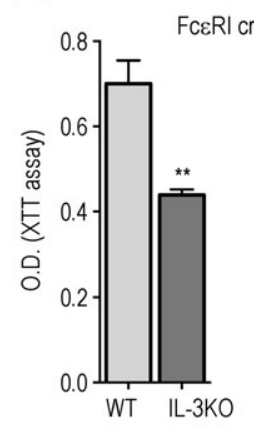

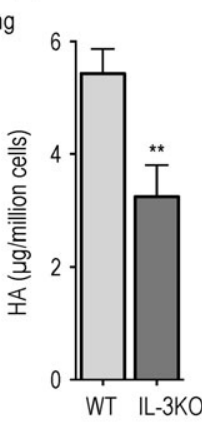

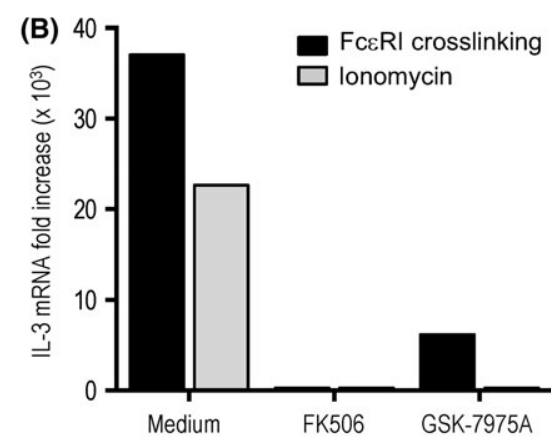

(E)

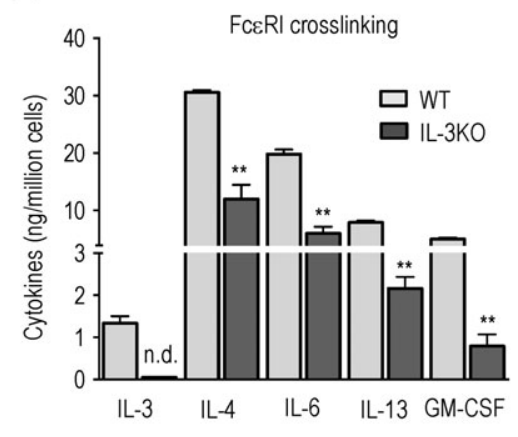

(G)

(F)

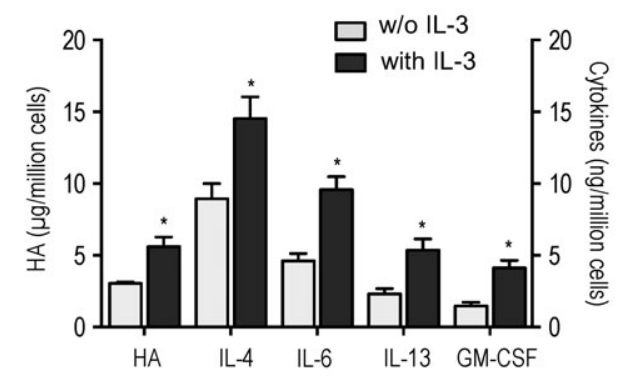

FIGURE 2 Autocrine IL-3 loop generated by IL-3-derived basophils upon FceRI crosslinking. A, IL-3 production in response to DNP-HSA/anti-DNP IgE or ionomycin with or without FK506 or GSK$7975 \mathrm{~A}$, measured in culture supernatants from C57BL/6J mice after a 20-h incubation $(n=3)$. B, IL-3 mRNA expression assessed by quantitative RT-PCR in IL-3 derived basophils from C57BL/6J mice stimulated for $4 \mathrm{~h}$. Values are reported as fold increase in mRNA levels relative to those obtained with IL-3-derived basophils before stimulation. Data are representative of three experiments. C, Viability of basophils from C57BL/10 WT and IL-3deficient (IL-3KO) mice following $20 \mathrm{~h}$ of stimulation, as measured by the XTT assay $(n=6)$. (D and E), HA and cytokines production by IL-3-derived basophils from WT $(n=4)$ and IL-3KO mice $(n=8)$ following Fc\&RI crosslinking. $P$ values were determined between WT and IL-3KO mice. F, HA and cytokine production by IL-3derived basophils from IL-3KO mice $(n=4)$ after Fc\&RI crosslinking in the presence or absence of exogenous IL-3. G, Viability of basophils from WT and IL-3KO mice following 20 h of stimulation $(n=4)$ without or with IL-3, measured by the XTT assay. $P$ values were determined between cells cultured with or without IL-3
(Figure 3B). By contrast, IL-13 and GM-CSF were produced at similarly low levels and there was no significant difference in histamine synthesis (Figure 3B).

These quantitative differences were associated with qualitative changes, which rendered the Fc\&RI-induced production of histamine and cytokines by IL-3-derived basophils significantly more sensitive to the inhibition of calcium signaling by the calcineurin inhibitor FK506, relative to their ex vivo BMRB counterpart (Figure 3C). A similar conclusion applies to the SOC inhibitor GSK-7975A that abrogated the effect of ionomycin in both basophil types, but inhibited the response to IL-3 or Fc\&RI crosslinking more effectively in IL3-derived basophils, as compared to BMRB (Table S4).

Furthermore, only IL-3-derived basophils produced substantial amounts of IL-3 in response to FceRI crosslinking or ionomycin (Figure 3D). In accordance with this finding, no IL-3 transcripts were detected in BMRB, whether they were stimulated through Fc\&RI or ionomycin (data not shown), while high levels of IL-3 mRNA were readily expressed in IL-3-derived basophils in the same conditions (Figure 2B). These data indicate that BMRB and IL-3-derived basophils are functionally distinct.

\section{4 | Treatment of ex vivo-sorted medullary basophils with IL-3 promotes endogenous IL-3 production and enhances histamine and cytokine synthesis in response to Fc\&RI crosslinking}

Basophils generated from hematopoietic progenitors in the presence of IL-3 are more reactive to Fc\&RI stimulation than their BMRB counterpart, raising the question whether these populations belong to two distinct subsets or two different stages of maturation. To address this issue, $C D 49 b^{+} F c \varepsilon R I^{+} c-k i t^{-} C D 11 b^{+}$BMRB were electronically sorted, incubated with $\mathrm{IL}-3$, and compared with freshly isolated BMRB for their response to Fc\&RI stimulation. Exposure to IL-3 yielded a population that behaved like IL-3-derived basophils as its response to $\mathrm{F} c \varepsilon \mathrm{RI}$ crosslinking was strikingly increased in terms of histamine and IL-4 secretion, concomitantly with IL-3, IL13 , and GM-CSF, conversely to the initial population (Figure 4). In addition, this pretreatment rendered histamine and cytokine production highly sensitive to the blockade of calcineurin signaling by FK506 (Figure 4), a characteristic shared with IL-3-derived basophils (Table S3). 
FIGURE 3 Functional distinctions between BMRB and IL-3-derived basophils. $\mathrm{HA}$ and cytokines were measured in supernatants from BMRB $(\mathrm{n}=8)$ and IL-3derived basophils $(n=27)$ from $C 57 B L / 6$ J mice in response to $\mathrm{A}$, Fc\&RI crosslinking or $B$, IL-3. $P$ values were determined between BMRB and IL-3-derived basophils. C, Percent inhibition of HA and cytokine production in response to Fc\&RI crosslinking by FK506 $(\mathrm{n}=8)$. D, IL-3 measured in supernatants of IL-3-derived basophils or BMRB after Fc\&RI crosslinking or ionomycin stimulation $(\mathrm{n}=3)$
(A)

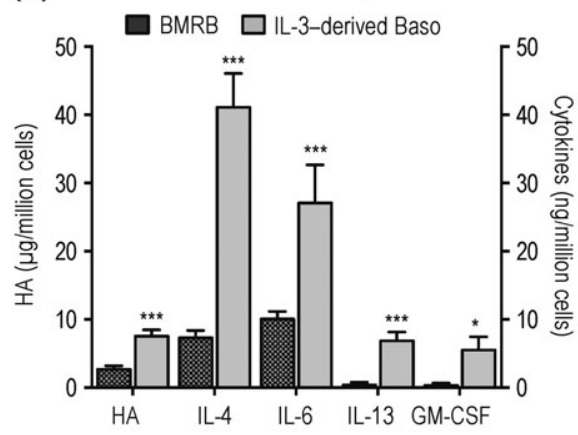

(C)

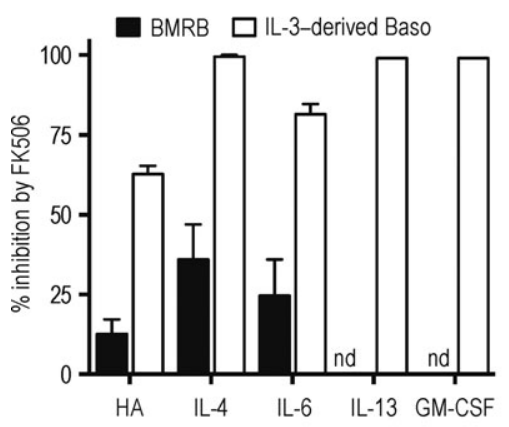

(B) IL-3 stimulation

BMRB $\square$ IL-3-derived Baso

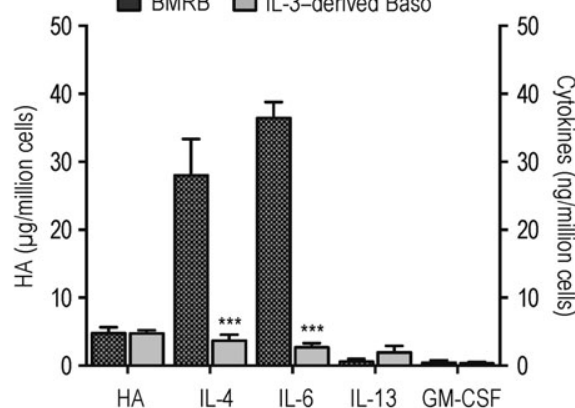

(D)

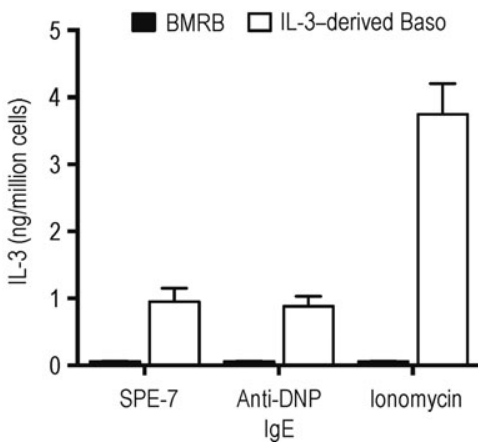

$\mathrm{IL}-4$
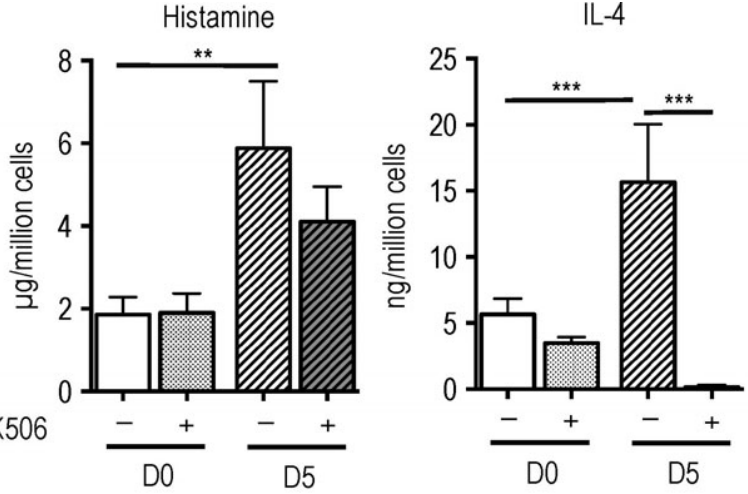

IL-3

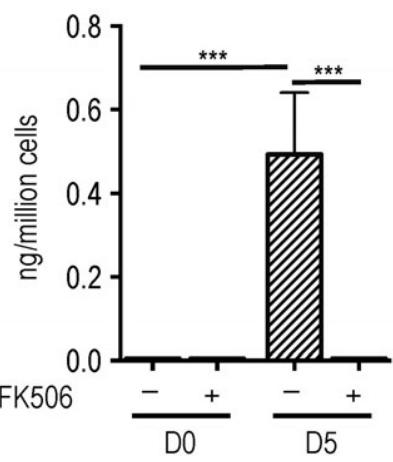

IL-13

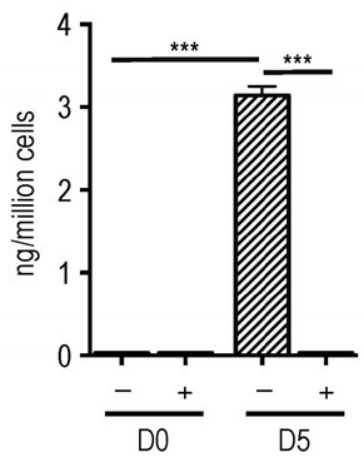

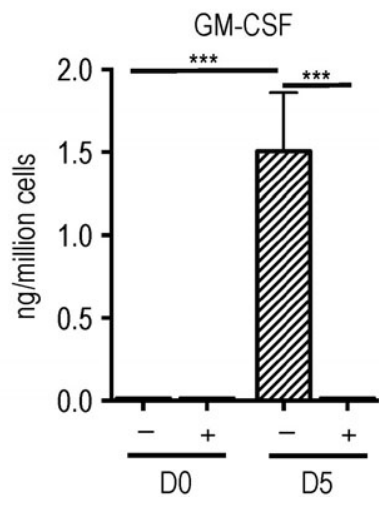

FIGURE 4 Functional transformation of $\mathrm{BMRB}$ in response to IL-3. Ex vivo-sorted BMRB from C57BL/6J mice were directly (D0) $(\mathrm{n}=3)$ stimulated with DNP-HSA/ anti-DNP IgE with or without FK506 or cultured during 5 days (D5) ( $n=5)$ with IL3 before this stimulation. HA, IL-4, IL-13, GM-CSF, and IL-3 were then determined in culture supernatants. Results are expressed after subtraction of background production (culture medium alone)

\section{5 | Basophils recovered from mice injected with IL-3 respond more efficiently to Fc\&RI crosslinking}

Further, we explored the in vivo response of basophils to IL-3. It has been established that in vivo basophil expansion can be greatly increased when IL-3 is injected as a complex with anti-IL-
$3 \mathrm{mAb}$, which stabilizes and prolongs the activity of the growth factor. ${ }^{34,35}$ Using this approach, we found that IL-3 plus anti-IL-3 injection increased not only the number of medullary, splenic, liver, and lung basophils (Figure 5A), but also enhanced their histamine and cytokine synthesis in response to FceRI crosslinking (Figure 5B,C). 
(A)

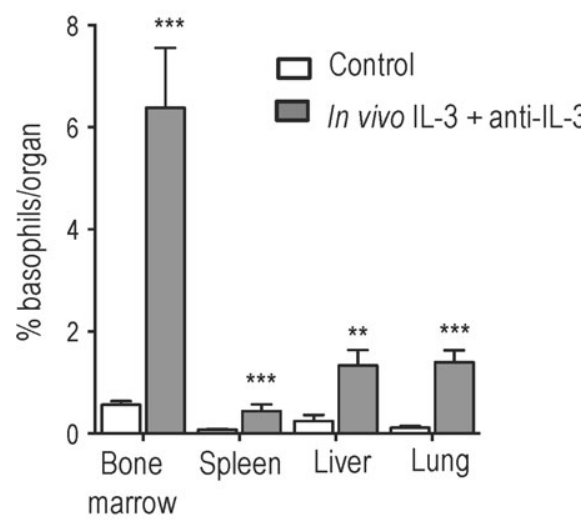

(C)

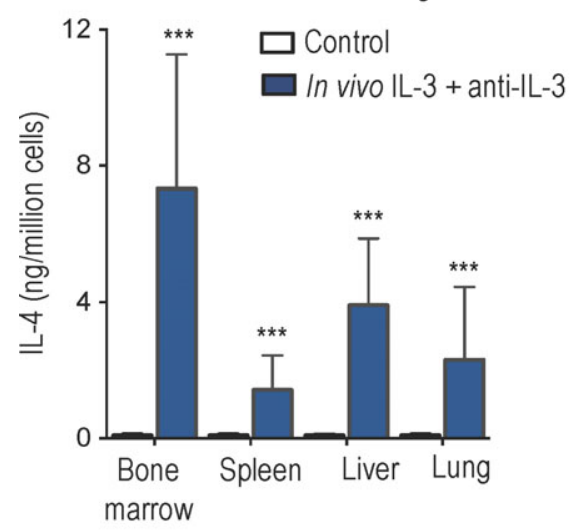

(B)

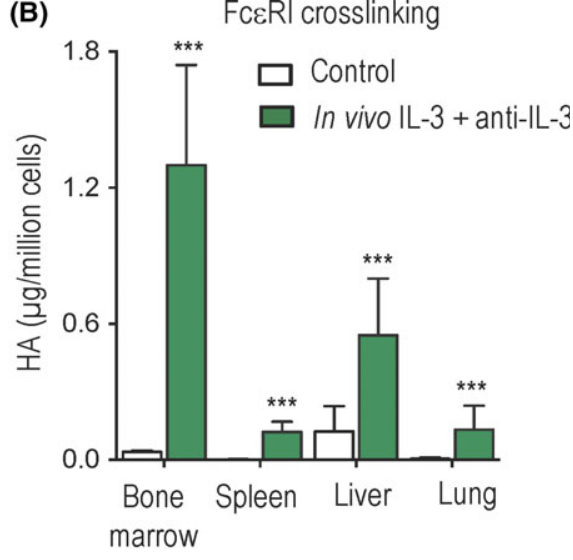

(D)

FcERI crosslinking

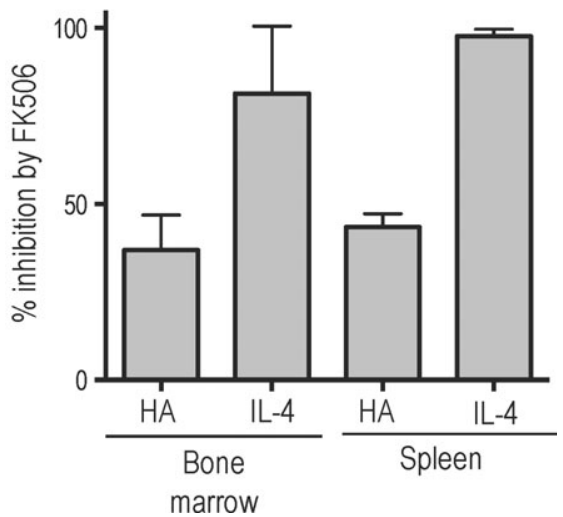

FIGURE 5 Effect of in vivo IL-3 injection on basophil functions. Comparison between basophils recovered from C57BL/ 6J mice injected with saline (control) or IL$3+$ anti-IL-3 complexes. A, Frequency of basophils per organ identified as Fc\&RI ${ }^{+} C D 49 b^{+}$cells $(n=7)$. Levels of (B) HA or (C) IL-4 produced in response to Fc\&RI stimulation in $\mu \mathrm{g}$ or $\mathrm{ng} / \mathrm{million}$ cells, respectively $(n=7)$. D, Percent inhibition of HA and IL-4 production by FK506 following FceRI crosslinking on bone marrow or spleen cells from IL-3 + anti-IL-3 injected mice $(\mathrm{n}=3)$ [Colour figure can be viewed at wileyonlinelibrary.com]
Similar to IL-3-derived basophils, the population expanded in vivo was highly sensitive to the inhibition of histamine and IL-4 production by the calcineurin inhibitor FK506 (Figure 5D), relative to its untreated counterpart (Figure $3 \mathrm{C}$ ). Finally, bone marrow cells from IL-3-injected mice produced detectable amounts of IL-13 $\left(574 \pm 229 \mathrm{pg} / 10^{6}\right.$ cells; $\left.n=4\right)$ and GM-CSF $\left(859 \pm 484 \mathrm{pg} / 10^{6}\right.$ cells; $n=4$ ) in response to $\mathrm{F} c \varepsilon R I$ stimulation, while neither cytokine was detected in supernatants from control mice.

\section{6 | IL-3-derived basophils are required for AHR}

Having established that fully competent basophils capable of autocrine IL-3 production can emerge either in vitro or in vivo following IL-3 treatment, we addressed the relevance of this finding in a pathophysiological situation using a murine model of allergic asthma. ${ }^{36,37}$ Basophils were present in BALF (bronchoalveolar lavage fluid) from ovalbumin (OVA)-induced asthmatic mice (Figure 6A and Figure S5A), confirming our previous data. ${ }^{38}$ They expressed Fc\&RI, CD200R3, and CD69 (Figure S5A). Basophils recovered from BALF secreted both IL-4 and IL-13 following FcER1 crosslinking (Figure 6B), indicating that they behave like IL-3-derived basophils rather than BMRB. In the same line of evidence, BALF cells from asthmatic mice enhanced their expression of mRNA encoding GM-CSF, IL-13, and IL-3 when activated in vitro by FceRI crosslinking (Figure S5B).
Using IL-3-deficient mice, we examined whether IL-3 and basophils could play a role in the development of allergic asthma symptoms. We found that the airway eosinophilia displayed by OVA-sensitized and OVA-challenged IL-3KO mice was similar to wild-type (WT) controls (Figure 6C). Likewise, the number of basophils in BALF was increased in both cases (Figure 6D), and the percentage of monocytes, eosinophils, neutrophils, or lymphocytes was also not significantly different between the two strains (Figure S6A). Lastly, total IgE as well as specific anti-OVA IgE levels in the serum of IL-3KO and WT asthmatic mice was also similar (Figure S6B).

Yet, despite these common features, OVA-sensitized and OVAchallenged IL-3KO mice displayed no detectable airway hyperreactivity (AHR) in contrast to their WT counterpart (Figure 6E). Furthermore, the frequency of viable basophils and of IL- $4^{+}$and IL- $13^{+}$cells among gated lung basophils from IL-3KO mice was reduced compared to WT controls (Figure S6C), showing that in the absence of IL-3 basophil survival is compromised, as is their pro-Th2 cytokine production. These findings led to the question whether AHR could be restored by transferring fully competent IL-3-derived basophils to OVA-treated $\mathrm{IL}-3 \mathrm{KO}$ mice. As shown in Figure $6 \mathrm{E}$, this was effectively the case. In stark contrast, adoptive transfer of IL-3-derived basophils from IL$3 \mathrm{KO}$ mice had no effect (Figure 6E). The failure to develop an effective AHR response in IL-3-deficient mice and the inability of IL-3derived basophils from IL-3KO mice to restore the AHR can therefore 
FIGURE 6 Contribution of IL-3dependent basophils to allergic airway hyperreactivity. A, Basophil counts in BALF of asthmatic (OVA-immunized and OVAchallenged, $n=29$ ) BALB/c mice compared to controls $(\mathrm{NaCl}, \mathrm{n}=19)$. $\mathrm{B}$, Lung basophils from asthmatic BALB/c mice were stimulated with anti-DNP IgE or medium during $6 \mathrm{~h}$. Both IL-4 and IL-13 were detected by intracellular staining among basophils gated as

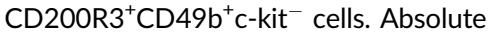
numbers of eosinophils (C) or basophils (D) in BALF of IL-3KO and wild-type (WT) asthmatic (OVA) or nonasthmatic ( $\mathrm{NaCl})$ mice. E, Lung resistance (Rtr) was measured $24 \mathrm{~h}$ after the last $\mathrm{NaCl}$ or OVA challenge in WT ( $n=9$ for OVA; 6 for $\mathrm{NaCl}$ ), IL-3KO ( $\mathrm{n}=9$ ), and IL-3KO mice having received IL-3-derived basophils from WT $(n=3)$ or IL-3KO $(n=3)$ mice. The AHR values were analyzed with repeated-measures 2-way ANOVA followed by Bonferroni correction as a post hoc test. $P$ values were determined between OVA WT and OVA IL-3KO mice $(* P<.05, P<.01 * *)$, between OVA IL$3 K O$ and OVA IL-3KO mice after adoptive transfer of IL-3-derived basophils from IL3KO mice (\#P $<.05, P<.01 \# \#)$, and between OVA IL-3KO after adoptive transfer of IL-3-derived basophils from WT or IL-3KO mice $(\wedge P<.05, \wedge \wedge P<.01)$ [Colour figure can be viewed at wileyonlinelibrary.com]
(A)

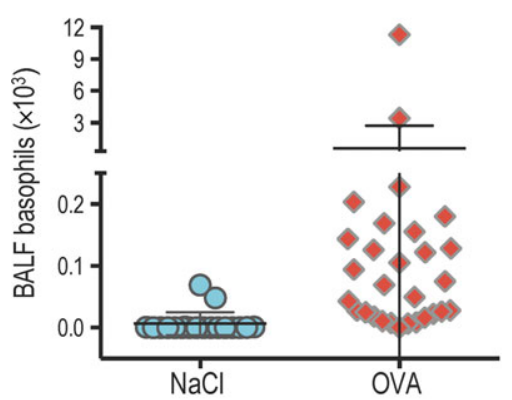

(C)

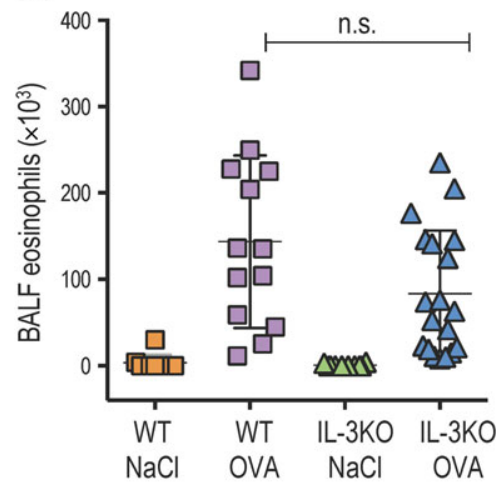

(B)

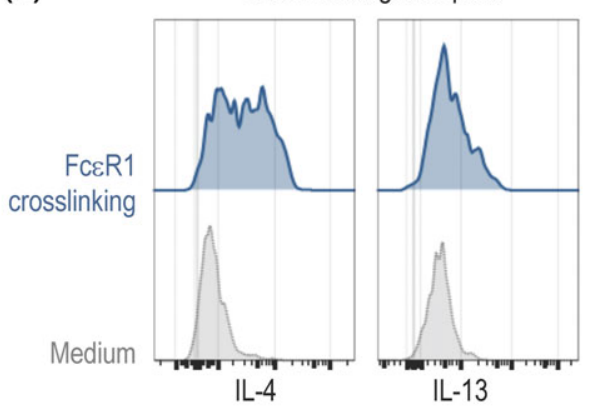

(D)

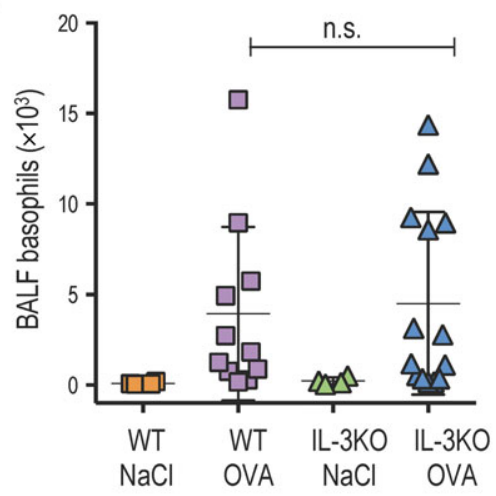

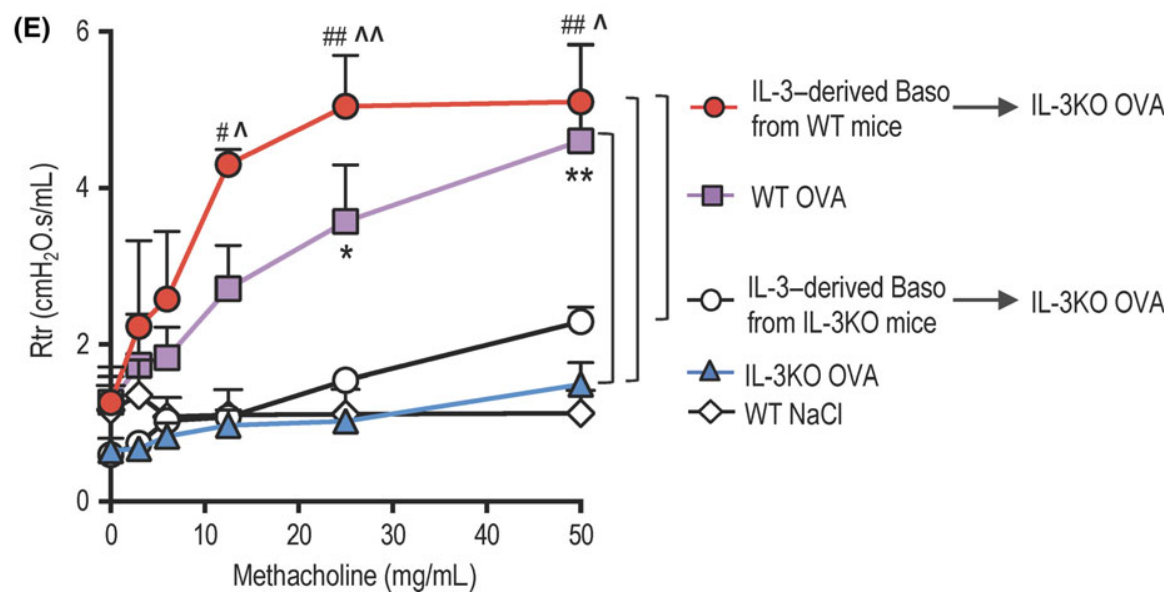

be ascribed entirely to the lack of IL-3 production by basophils, which compromises their contribution to asthmatic symptoms. The growth factor deficiency explains likewise the two other shortcomings of basophils from IL-3-deficient mice, namely their limited capacity to secrete $\mathrm{HA}$ and pro-inflammatory cytokines and their impaired survival upon FceRI crosslinking (Figure 2C-E). Hence, basophils depend on priming by their endogenous IL-3 to become competent in a model of allergic asthma in which they exert a deleterious effect by promoting AHR

\section{4 | DISCUSSION}

The present study provides evidence for a new role of exogenous and endogenous IL-3, namely the priming of bone marrow basophils to improve their responsiveness to Fc\&RI stimulation, which results in a more effective production of histamine, IL-4, IL-6, IL-13, and GM-CSF. This enhanced activity requires calcium flux and is dependent on calcineurin and store-operated calcium channels (SOCs). Exposure to IL-3 triggers an autocrine loop, which enables basophils to produce IL-3 as an endogenous growth factor, for optimal activation, survival, and in vivo functions. Indeed, IL-3-deficient mice fail to develop AHR in an experimental asthma model, while these respiratory symptoms can be restored upon adoptive transfer of IL-3derived basophils from WT but not from IL-3KO mice. From these findings, we conclude that the autocrine IL-3 loop endows basophils with the capacity to respond more efficiently to Fc\&RI stimulation, so as to induce AHR symptoms in asthmatic mice.

Our results demonstrate that basophils can adopt distinct functional characteristics determined by IL- 3 exposure and production. BMRB activities are barely diminished when intracellular calcium signaling is blocked, which is in stark contrast with those generated by 
basophils expanded in vitro or in vivo in the presence of IL-3. Calcium signaling is essential for diverse biological processes, and its intracellular concentration is highly controlled by specific mechanisms. ${ }^{29}$ Sustained calcium entry is obligatory for essentially all responses initiated through $\mathrm{T}, \mathrm{B}$, and Fc receptors, including proliferation and cytokine production. ${ }^{39}$ IL-3-derived basophils not only synthesized more histamine and cytokines in response to Fc\&RI stimulation, but also depend largely on calcineurin- and store-operated calcium channels (SOC) for their enhanced responsiveness. Even though mechanisms accounting for the regulation of mast cell functions through calcium signaling have been extensively investigated, no such studies have been carried out for basophils. Here, we show that BMRB and IL-3-derived basophils are differentially regulated by calcium-dependent mechanisms. After exposure to IL-3, basophil activities become highly dependent on calcineurin and SOCs and can generate their own IL-3, which acts in an autocrine fashion to increase histamine and cytokine production as well as survival in response to $\mathrm{F} c \varepsilon R \mathrm{R}$ crosslinking.

Previous reports have established that T lymphocyte-dependent contact hypersensitivity responses to haptens are impaired in IL-3deficient mice, which display attenuated basophil responses to nematode infection that result in compromised worm expulsion and increased resistance to blood-stage malaria. ${ }^{18,40-43}$ Our study is the first to examine the effect of IL-3 deficiency on the development of allergic asthmatic symptoms. It revealed a similar increase in eosinophil and basophil counts in BALF from OVA-treated IL-3KO and WT mice, as compared to untreated controls. Systemic allergic Th2 immune responses occurred in both IL-3-deficient and WT strains, as assessed by the high levels of circulating total and OVA specific antiIgE antibodies. It can therefore be concluded that these modifications occur independently from IL-3 and IL-3-deficient basophils. Nonetheless, OVA-treated IL-3KO mice were unable to develop AHR, which could be restored by adoptive transfer of IL-3-derived basophils from WT mice but not by their counterpart prepared from IL-3-deficient mice. This result established the requirement of the autocrine IL-3 loop to generate fully competent basophils that are critical for the severity of AHR in our model. Cytokines (IL-4, IL-6, IL-13, and GMCSF), histamine, and survival are impaired in IL-3-derived basophils from IL-3KO compared to WT mice, revealing a new facet of this cytokine with respect to basophil functions. These results are consistent with the assumption that basophils might have an important role during specific phases of allergic asthma. ${ }^{44}$ Although some differences exist between mouse and human basophils, a similar role for human basophils is supported by the fact that basophils were found in the airways of postmortem cases of fatal asthma. ${ }^{45}$

In conclusion, our findings indicate that BMRB could represent late basophil precursors with limited proliferation capacity having already acquired the typical surface markers and morphology of the basophil lineage. This would imply that basophils leave the bone marrow in a "naïv" state. In the periphery, IL-3 signaling enables these basophils to fully express their inherent Th2-like functions in response to the high-affinity receptor for IgE by producing IL-3 endogenously, setting off an autocrine amplification loop, which is critical for the onset of AHR. These newly acquired IL-3-dependent functions of basophils are finely regulated by calcium flux and SOCs. This new insight may aid in designing strategies to modulate these signaling pathways in vivo, so as to prevent basophil activation in specific asthmatic disease processes.

\section{ACKNOWLEDGMENTS}

We are indebted to Malcolm Begg (GlaxoSmithKline, UK) and Victor Tybulewicz (Medical Research Council) for providing GSK-7975A and IL-3KO mice, respectively. We thank C. Dietrich and E. Panafieu for technical assistance. This work was supported by grants from CNRS, INSERM, Université Paris Descartes, and Fondation Day Solvay.

\section{CONFLICT OF INTEREST}

The authors declare that they have no conflict of interests.

\section{AUTHOR CONTRIBUTIONS}

R.R.B., M.L.M, and M.D. conceived and designed the experiments. R.R.B and F.M. performed the research. R.R.B, F.M., E.S., M.L.M, and M.D. analyzed the data. S.M. and O.H. contributed with reagents, materials, or analysis tools. E.S., M.L.M, and M.D. wrote the manuscript.

\section{ORCID}

M. Leite-de-Moraes (iD http://orcid.org/0000-0002-2891-2269

\section{REFERENCES}

1. Schneider E, Thieblemont N, De Moraes ML, Dy M. Basophils: new players in the cytokine network. Eur Cytokine Netw. 2010;21:142153.

2. Sullivan BM, Locksley RM. Basophils: a nonredundant contributor to host immunity. Immunity. 2009;30:12-20.

3. Chirumbolo S. State-of-the-art review about basophil research in immunology and allergy: is the time right to treat these cells with the respect they deserve? Blood Transfus. 2012;10:148-164.

4. Stone KD, Prussin C, Metcalfe DD. IgE, mast cells, basophils, and eosinophils. J Allergy Clin Immunol. 2010;125:S73-S80.

5. Voehringer D. Protective and pathological roles of mast cells and basophils. Nat Rev Immunol. 2013;13:362-375.

6. Qi X, Nishida J, Chaves L, Ohmori K, Huang H. CCAAT/enhancerbinding protein alpha (C/EBPalpha) is critical for interleukin-4 expression in response to FcepsilonRI receptor cross-linking. J Biol Chem. 2011;286:16063-16073.

7. Sloane DE, Tedla N, Awoniyi M, et al. Leukocyte immunoglobulinlike receptors: novel innate receptors for human basophil activation and inhibition. Blood. 2004;104:2832-2839.

8. Kojima T, Obata K, Mukai K, et al. Mast cells and basophils are selectively activated in vitro and in vivo through CD200R3 in an IgEindependent manner. J Immunol. 2007;179:7093-7100.

9. Yamanishi $\mathrm{Y}$, Miyake K, Iki M, Tsutsui H, Karasuyama H. Recent advances in understanding basophil-mediated Th2 immune responses. Immunol Rev. 2017;278:237-245.

10. Mitre E, Taylor RT, Kubofcik J, Nutman TB. Parasite antigen-driven basophils are a major source of IL-4 in human filarial infections. J Immunol. 2004;172:2439-2445. 
11. Sokol CL, Chu NQ, Yu S, Nish SA, Laufer TM, Medzhitov R. Basophils function as antigen-presenting cells for an allergen-induced $T$ helper type 2 response. Nat Immunol. 2009;10:713-720.

12. Perrigoue JG, Saenz SA, Siracusa MC, et al. MHC class II-dependent basophil-CD4 + T cell interactions promote $T(H) 2$ cytokine-dependent immunity. Nat Immunol. 2009;10:697-705.

13. Yoshimoto T, Yasuda K, Tanaka H, et al. Basophils contribute to $T(H) 2-\lg E$ responses in vivo via IL-4 production and presentation of peptide-MHC class II complexes to CD4 + T cells. Nat Immunol. 2009;10:706-712.

14. Motomura $\mathrm{Y}$, Morita $\mathrm{H}$, Moro $\mathrm{K}$, et al. Basophil-derived interleukin-4 controls the function of natural helper cells, a member of ILC2s, in lung inflammation. Immunity. 2014;40:758-771.

15. Schneider E, Machavoine F, Pleau JM, et al. Organic cation transporter 3 modulates murine basophil functions by controlling intracellular histamine levels. J Exp Med. 2005;202:387-393.

16. Schneider E, Machavoine F, Bricard-Rignault R, et al. Downregulation of basophil-derived IL-4 and in vivo $\mathrm{T}(\mathrm{H}) 2$ IgE responses by serotonin and other organic cation transporter 3 ligands. J Allergy Clin Immunol. 2011;128:864-871.

17. Oetjen LK, Noti M, Kim BS. New insights into basophil heterogeneity. Semin Immunopathol. 2016;38:549-561.

18. Lantz CS, Boesiger J, Song $\mathrm{CH}$, et al. Role for interleukin-3 in mastcell and basophil development and in immunity to parasites. Nature. 1998;392:90-93.

19. Schwartz C, Eberle JU, Hoyler T, Diefenbach A, Lechmann M, Voehringer D. Opposing functions of thymic stromal lymphopoietinresponsive basophils and dendritic cells in a mouse model of atopic dermatitis. J Allergy Clin Immunol. 2016;138:1443-1446.

20. Siracusa MC, Saenz SA, Hill DA, et al. TSLP promotes interleukin3 -independent basophil haematopoiesis and type 2 inflammation. Nature. 2011;477:229-233.

21. Salabert-Le Guen N, Hemont C, Delbove A, et al. Thymic stromal lymphopoietin does not activate human basophils. J Allergy Clin Immunol. 2018;141:1476-1479.

22. Schneider E, Pollard H, Lepault F, Guy-Grand D, Minkowski M, Dy M. Histamine-producing cell-stimulating activity. Interleukin 3 and granulocyte-macrophage colony-stimulating factor induce de novo synthesis of histidine decarboxylase in hemopoietic progenitor cells. J Immunol. 1987;139:3710-3717.

23. Schneider E, Petit-Bertron AF, Bricard R, et al. IL-33 activates unprimed murine basophils directly in vitro and induces their in vivo expansion indirectly by promoting hematopoietic growth factor production. J Immunol. 2009;183:3591-3597.

24. Michel ML, Lenoir C, Massot B, et al. SLAM-associated protein favors the development of iNKT2 over iNKT17 cells. Eur J Immunol. 2016;46:2162-2174.

25. Massot B, Michel ML, Diem S, et al. TLR-induced cytokines promote effective proinflammatory natural Th17 cell responses. J Immunol. 2014;192:5635-5642.

26. Bonner JM, Boulianne GL. Diverse structures, functions and uses of FK506 binding proteins. Cell Signal. 2017;38:97-105.

27. Liu J, Farmer JD Jr, Lane WS, Friedman J, Weissman I, Schreiber SL. Calcineurin is a common target of cyclophilin-cyclosporin A and FKBP-FK506 complexes. Cell. 1991;66:807-815.

28. Thastrup O, Cullen PJ, Drobak BK, Hanley MR, Dawson AP. Thapsigargin, a tumor promoter, discharges intracellular $\mathrm{Ca} 2+$ stores by specific inhibition of the endoplasmic reticulum Ca2(+)-ATPase. Proc Natl Acad Sci U S A. 1990;87:2466-2470.

29. Hogan PG, Lewis RS, Rao A. Molecular basis of calcium signaling in lymphocytes: STIM and ORAI. Annu Rev Immunol. 2010;28:491-533.

30. Yeung PS, Yamashita M, Prakriya M. Pore opening mechanism of CRAC channels. Cell Calcium. 2017;63:14-19.

31. Derler I, Schindl R, Fritsch R, et al. The action of selective CRAC channel blockers is affected by the Orai pore geometry. Cell Calcium. 2013;53:139-151.
32. Ashmole I, Duffy SM, Leyland ML, Morrison VS, Begg M, Bradding P. CRACM/Orai ion channel expression and function in human lung mast cells. J Allergy Clin Immunol. 2012;129:1628-1635.

33. Rice LV, Bax HJ, Russell LJ, et al. Characterization of selective calcium-release activated calcium channel blockers in mast cells and T-cells from human, rat, mouse and guinea-pig preparations. Eur J Pharmacol. 2013;704:49-57.

34. Finkelman FD, Madden KB, Morris SC, et al. Anti-cytokine antibodies as carrier proteins. Prolongation of in vivo effects of exogenous cytokines by injection of cytokine-anti-cytokine antibody complexes. J Immunol. 1993;151:1235-1244.

35. Ohmori K, Luo Y, Jia Y, et al. IL-3 induces basophil expansion in vivo by directing granulocyte-monocyte progenitors to differentiate into basophil lineage-restricted progenitors in the bone marrow and by increasing the number of basophil/mast cell progenitors in the spleen. J Immunol. 2009;182:2835-2841.

36. Lisbonne M, Diem S, de Castro Keller A, et al. Cutting edge: invariant $\mathrm{V}$ alpha 14 NKT cells are required for allergen-induced airway inflammation and hyperreactivity in an experimental asthma model. J Immunol. 2003;171:1637-1641.

37. Hachem P, Lisbonne M, Michel ML, et al. Alpha-galactosylceramideinduced iNKT cells suppress experimental allergic asthma in sensitized mice: role of IFN-gamma. Eur J Immunol. 2005;35:2793-2802.

38. Ramadan A, Van Pham L, Machavoine F, et al. Activation of basophils by the double-stranded RNA poly(A:U) exacerbates allergic inflammation. Allergy. 2013;68:732-738.

39. Di Capite J, Parekh AB. CRAC channels and $\mathrm{Ca} 2+$ signaling in mast cells. Immunol Rev. 2009;231:45-58.

40. Mach N, Lantz CS, Galli SJ, et al. Involvement of interleukin-3 in delayed-type hypersensitivity. Blood. 1998;91:778-783.

41. Gillessen S, Mach N, Small C, Mihm M, Dranoff G. Overlapping roles for granulocyte-macrophage colony-stimulating factor and interleukin-3 in eosinophil homeostasis and contact hypersensitivity. Blood. 2001;97:922-928.

42. Kimura K, Song CH, Rastogi A, Dranoff G, Galli SJ, Lantz CS. Interleukin-3 and c-Kit/stem cell factor are required for normal eosinophil responses in mice infected with Strongyloides venezuelensis. Lab Invest. 2006;86:987-996.

43. Auclair SR, Roth KE, Saunders BL, et al. Interleukin-3-deficient mice have increased resistance to blood-stage malaria. Infect Immun. 2014;82:1308-1314.

44. Lichtenstein LM, Bochner BS. The role of basophils in asthma. Ann N Y Acad Sci. 1991;629:48-61.

45. Koshino T, Teshima S, Fukushima N, et al. Identification of basophils by immunohistochemistry in the airways of post-mortem cases of fatal asthma. Clin Exp Allergy. 1993;23:919-925.

\section{SUPPORTING INFORMATION}

Additional supporting information may be found online in the Supporting Information section at the end of the article.

How to cite this article: Rignault-Bricard R, Machavoine F, Mecheri S, et al. IL-3-producing basophils are required to exacerbate airway hyperresponsiveness in a murine inflammatory model. Allergy. 2018;73:2342-2351. https://doi.org/10.1111/all.13480 